A domain decomposition method for approximating the conformal modules of

long quadrilaterals

N. Papamichael and N.S. Stylianopoulos 
BRUNEL UNIVERSITY SEP 1991

\section{LIBRARY}

w9199049 


\title{
A Domain Decomposition Method \\ for Approximating the Conformal Modules of Long Quadrilaterals
}

N. Papamichael $*$ and N.S. Stylianopoulos *

\begin{abstract}
This paper is concerned with the study of a domain decomposition method for approximating the conformal modules of long quadrilaterals. The method has been studied already by us and also by D Gaier and W K Hayman, but only in connection with a special class of quadrilaterals, viz. quadrilaterals where: (a) the defining domain is bounded by two parallel straight lines and two Jordan arcs, and (b) the four specified boundary points are the four corners where the arcs meet the straight lines.

Our main purpose here is to explain how the method may be extended to a wider class of quadrilaterals than that indicated above.
\end{abstract}

Keywords: Conformal mapping, quadrilaterals, conformal modules, domain decomposition

* Department of Mathematics and Statistics, Brunei University, Uxbridge, Middlesex UB8 3PH, U.K. 



\section{Introduction}

Let $\Omega$ be a simply-connected domain in the complex z-plane $(z=x+i y)$, and consider a system consisting of $\Omega$ and four distinct points $z_{1}, z_{2}, z_{3}, z_{4}$ in counterclockwise order on its boundary $\partial \Omega$ Such a system is said to be a quadrilateral $Q$ and is denoted by

$$
Q:=\left\{\Omega ; z_{1}, z_{2}, z_{3}, z_{4}\right\}
$$

The conformal module $m(Q)$ of $Q$ is defined as follows:

Let $R_{H}$ be a rectangle of the form

$$
R_{H}:=\{(\xi, \eta): 0<\xi<1,0<\eta<H\},
$$

in the $w$-plane ( $W=\xi+\mathrm{i} \eta$ ). Then, $\mathrm{m}(Q)$ is the unique value of $H$ for which $Q$ is conformally equivalent to the rectangular quadrilateral

$$
Q^{\prime}:=\left\{R_{H} ; 0,1,1+i H, i H\right\}
$$

By this we mean that for $H=m(Q)$ and for this value only there exists a unique conformal map $\Omega \rightarrow R_{H}$, which takes the four points $\mathrm{z}_{1}, \mathrm{z}_{2}, \mathrm{z}_{3}, \mathrm{z}_{4}$ respectively onto the four corners $0,1,1+i H, i H$ of $R_{H}$.

This paper is concerned with the study of a domain decomposition method (DDM) for computing approximations to the conformal modules of "long " quadrilaterals $Q$. This involves decomposing $Q$ into two or more component quadrilaterals $Q_{j}, j=1,2, \ldots$, and approximating $m(Q)$ by the sum $\sum m\left(Q_{j}\right)$ of the conformal modules of the component quadrilaterals. The method has been studied already by us $[9,10]$ and also by Gaier and Hayman $[2,3]$, but only in connection with a special class of quadrilaterals, viz. quadrilaterals $Q:=\left\{\Omega ; \mathrm{z}_{1}, \mathrm{z}_{2}, \mathrm{z}_{3}, \mathrm{z}_{4}\right\}$ where : (a) The domain $\Omega$ is bounded by two parallel straight lines $l_{1}$ ' $l_{2}$ 'and two Jordan $\operatorname{arcs} \gamma_{1}, \gamma_{2}$,. (b) The points $\mathrm{z}_{1}, \mathrm{z}_{2}, \mathrm{z}_{3}, \mathrm{z}_{4}$ are the four corners where the arcs $\gamma_{1}, \gamma_{2}$, meet the straight lines $l_{1}{ }^{\prime} l_{2}$.

Of the four references cited above, our two earlier papers $[9,10]$ are concerned with the problems of approximating both the conformal module $m(Q)$ and the full conformal map $R_{m(Q)} \rightarrow \Omega$ and include the following:

(i) Computable estimatesfor theerror in theDDM approximation to $m(Q)$, and also for various other errors connected with the DDM approximation to the conformal map $R_{m(Q) \rightarrow} \Omega$ These are derived in $[9, \S 4]$, by assuming that the two boundary arcs $\gamma_{1}, \gamma_{2}$, satisfy certain smoothness conditions.

(ii) A number of conjectures concerning the assumptions underwhich theerror estimates of [9] hold (cf. [10, §3]).

(iii) The results of several numerical examples that confirm the theory of [9] and support the conjectures made in $[10]$ (cf.[9,§5] and[10, §3]). 
The two more recent papers [2,3] by Gaier and Hayman are concerned only with the problem of approximating $m(Q)$, and contain several important results that enhance considerably the associated theory. In particular,[2,3]include the following:

(iv) An estimate for the error in the DDM approximation to $m(Q)$, which (unlike our estimate in [9])is derived without imposing any conditions on the boundary segments $\gamma_{1}$, $\gamma_{2}^{\prime}$ other than requiring that they are Jordan arcs. (This proves one of the conjectures made in [10].)

(v) Certain other estimates that connect the conformal modules of the quadrilaterals involved in the decomposition with the conformal radii of certain curves associated with die $\operatorname{arcs} \gamma_{1}{ }^{\prime} \gamma_{2}^{\prime}$

In this paper we consider again the problem of computing approximations to $m(Q)$,and investigate the possibility of extending the application of the DDM to a wider class of quadrilaterals than that studied in $[2,3,9,10]$. Our main objective is to show that the method does, indeed, have much wider applicability and, for each new application, to derive an estimate of the error in the resulting DDM approximation to $m(Q)$. We shall do this by making use of two new theorems, which are simple consequences of the results of Gaier and Hayman [2,3] mentioned in (iv) and (v) above.

The paper is organized as follows: In Section 2 we set up various notations and state, without proof, the results of [2,3]which are needed for our subsequent work. In Section 3we prove two new theorems concerning the decomposition of general quadrilaterals. These theorems are then used in Section 4 to identify several new types of quadrilaterals for which the DDM is applicable, and to derive estimates for the errors in the resulting approximations to $m(Q)$. Finally, in Section 5 we present three numerical examples that illustrate the much wider applicability of the method.

We end this introductory section by noting that the DDM is of considerable practical interest for the following two reasons: (a) The method can be used to overcome the "crowding" difficulties associated with the conventional approach of seeking to determine $m(Q)$ by going via the unit disc or the upper half plane (see $[8, \S 3.1]$ and the other references cited there ).(b)It is often possible to decompose a complicated quadrilateral into very simple component quadrilaterals ( see the examples in Section 5 ).

\section{Preliminary results and notations}

We shall use throughout the following notations :

(i) $\Omega$ and $Q:=\left\{\Omega ; \mathrm{Z}_{1}{ }^{\prime} \mathrm{Z}_{2}{ }^{\prime} \mathrm{Z}_{3}{ }^{\prime} \mathrm{Z}_{4}\right\}$ will denote respectively the original domain and the corresponding quadrilateral.

(ii) $\Omega_{1}, \Omega_{12}, \cdots$, and $Q_{1}{ }^{\prime} Q_{2}, \cdots$, will denote the principal subdomains and corresponding com-

ponent quadrilaterals of the decomposition under consideration. 
In addition, we shall use a multisubscript notation to denote the other subdomains and quadrilaterals that arise when the decomposition of $Q$ involves more than one crosscut. For example, consider the situation illustrated in Fig. 2.1, where the decomposition of

$$
\mathrm{Q}:=\left\{\Omega ; \mathrm{z}_{1} \mathrm{z}_{2} \mathrm{z}_{3} \mathrm{z}_{4}\right\}
$$

is defined by two non-intersecting $\operatorname{arcs} \gamma_{1}, \gamma_{2}$, that join respectively two distinct points $\mathrm{a}$ and $\mathrm{b}$ on the boundary segment $\left(\mathrm{z}_{2}, \mathrm{z}_{3}\right)$ to two distinct points $d$ and $c$ on the boundary segment $\left(\mathrm{z}_{4}, \mathrm{z}_{1}\right)$. These two arcs subdivide $\Omega$ into three non-intersecting subdomains which we denote by $\Omega_{1}{ }^{\prime} \Omega_{2}$ and $\Omega_{3}{ }^{\prime}$ In addition the $\operatorname{arc} \gamma_{1}$, subdivides $\Omega$ into $\Omega_{1}$ and another subdomain which we denote by $\Omega_{2,3}$ ie. we take

$$
\bar{\Omega}_{2,3}:=\bar{\Omega}_{2} \cup \bar{\Omega}_{3} .
$$

Similarly, we say that $\gamma_{2}$, subdivides $\Omega$ into $\Omega_{1,2}$ and $\Omega_{3}$ ie. we take

$$
\bar{\Omega}_{1,2}:=\bar{\Omega}_{1} \cup \bar{\Omega}_{2} .
$$

Finally, we use the notations $Q_{1}, Q_{2}, Q_{3}, Q_{1,2}$ and $Q_{2,3}$ to denote respectively the quadrilaterals corresponding to the subdomains $\Omega_{1}, \Omega_{2}, \Omega_{3}, \Omega_{1.2}$ and $\mathrm{ft}, \Omega_{2.3}$ ie.

and

$$
Q_{1}:=\left\{\Omega_{1} ; \mathrm{z}_{1} ; \mathrm{z}_{2} ; \mathrm{a}, \mathrm{d}\right\}, \mathrm{Q}_{2}:=\left\{\Omega_{2} ; \mathrm{d}, \mathrm{a}, \mathrm{b}, \mathrm{c}\right\}, \mathrm{Q}_{3}:=\left\{\Omega_{3} ; \mathrm{c}, \mathrm{b}, \mathrm{z}_{3}, \mathrm{z}_{4}\right\}
$$

$$
Q_{1,2}:=\left\{\Omega_{1,2} ; z_{1,} z_{2}, b, c\right\}, Q_{2,3}:=\left\{\Omega_{2,3} ; d, a, z_{3}, z_{4}\right\}
$$

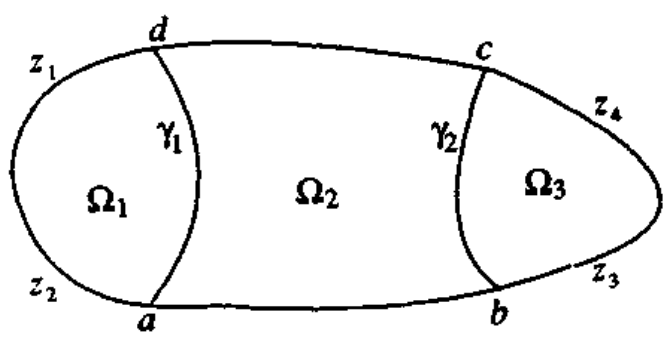

Figure 2.1

Let $\gamma$ be a Jordan arc that joins the boundary lines $x=0$ and $x=1$ of the strip

$$
\mathrm{S}:=\{z=x+i y: 0<\mathrm{x}<1\},
$$

and lies entirely within S, except for its two end points. In our work we shall refer frequently to the so-called exponential radius $r$ of such an arc $\gamma$. This is denned as follows ( cf. [2,§1.2] and see Fig. 2.2):

Let $\gamma^{*}$ be the arc obtained by translating $\gamma$ along the imaginary axis until it lies in $\operatorname{Im} \mathrm{z} \leq 0$, with at least one point on $\operatorname{Im} z=0$. Next, let $\Gamma^{*}$ be the image of $\gamma^{*}$ under the transformation $z \rightarrow \mathrm{e}^{\mathrm{i} \pi z}$, and let $\overline{\Gamma^{*}}$ denote the reflection of $\Gamma$ in the real axis. Finally, let $\Gamma$ denote the symmetric curve 
$\Gamma:=\Gamma^{*} \cup \overline{\Gamma^{*}}$ (Observe that $\Gamma$ surrounds the unit circle and meets the circle in at least two points; see Fig. 2.2.) Then, $r$ is defined to be the conformal radius of the interior of the curve $\Gamma$ with respect to the origin 0 . That is the exponential radius of the arc $\gamma$ is the radius of the disc $|W|<r$ onto which Int $\Gamma$ can be mapped by a conformal map $f$ such that $f(0)=0$ and $f^{\prime}(0)=1$.

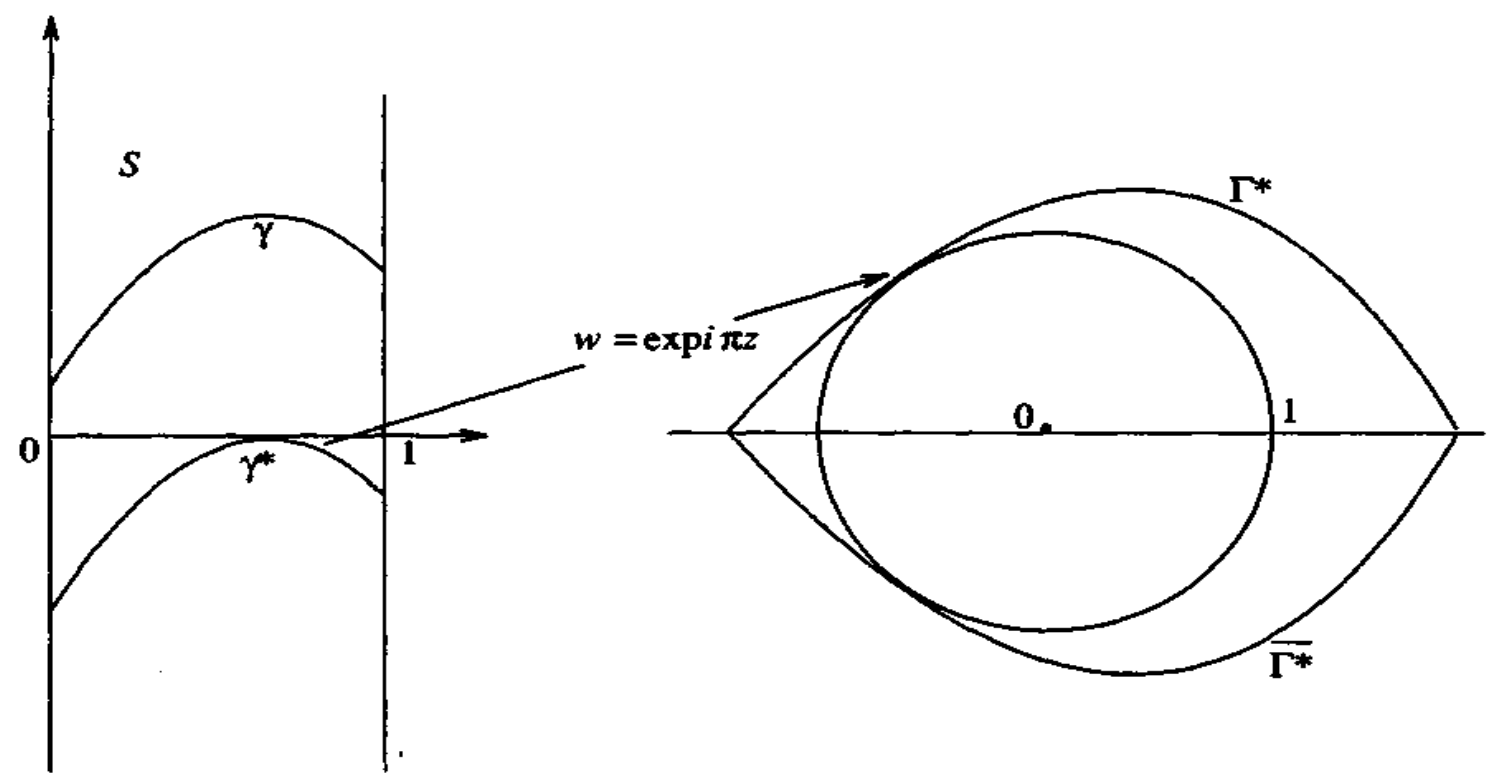

Figure 2.2

In the remainder of this section we state without proof various results which are needed for our subsequent work. These include, in particular, three theorems due to Gaier and Hayman [2, 3] which play a very crucial role in our work.

Consider a decomposition of the form illustrated in Fig. 2.3, where:

(i) The domain $\Omega$ of the original quadrilateral $Q:=\left\{\Omega, z_{1}, z_{2}, z_{3} . z_{4}\right\}$ is bounded by two segments of the lines $x=0$ and $x=1$, and two Jordan $\operatorname{arcs} \gamma_{1}, \gamma_{2}$.

(ii) The points $z_{1} z_{2}, z_{3}, z_{4}$ are the four corners where the arcs $\gamma_{1,} \gamma_{2}$ meet the lines $x=0$ and $x=1$.

(iii) The crosscut $l$ of subdivision is a straight line

$$
l:=\{(x, y): 0 \leq x \leq 1, y=c\},
$$

Parallel to the real axis, so that the component quadrilaterals are

$$
\mathrm{Q}_{1}:=\left\{\Omega_{1} ; \mathrm{z}_{1}, \mathrm{z}_{2}, 1+\mathrm{ic}, \mathrm{ic}\right\} \text { and } \mathrm{Q}_{2}:=\left\{\Omega_{2} ; \mathrm{ic}, 1+\mathrm{ic}, \mathrm{z}_{3,} \mathrm{z}_{4}\right\} \text {. }
$$


Let

$$
h_{1}:=c-\max \left\{\operatorname{Im} \mathrm{z}: \mathrm{z} \in \gamma_{1},\right\} \text { and } h_{2}:=\min \left\{\operatorname{Im} \mathrm{z}: \mathrm{z} \in \gamma_{2}\right\}-\mathrm{c} \text {, }
$$

as illustrated in Fig.2.3, and set

$$
h_{12}:=h_{1}+h_{2} \text { and } h:=\min \left(h_{1}, h_{2}\right) \text {, }
$$

Also, let $r_{l}$ and $r_{2}$ denote respectively the exponential radii of the arcs $\gamma_{1}$, and $\overline{\gamma_{2}}$. (Here, and

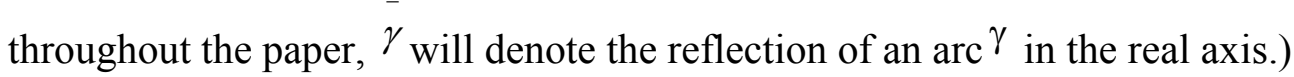

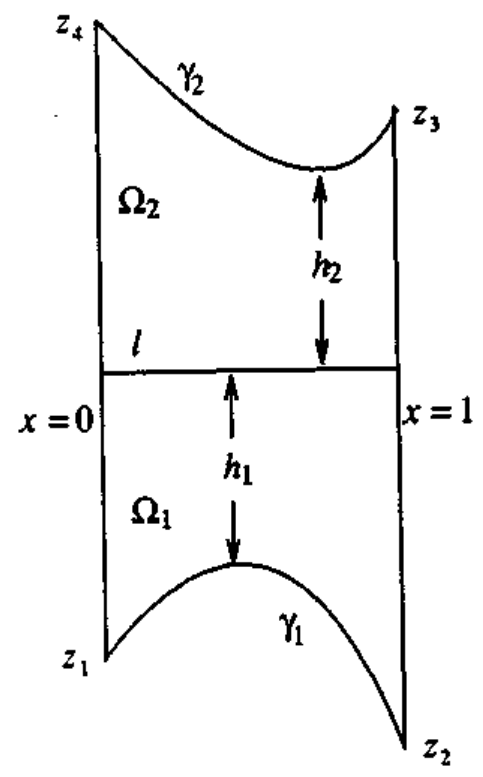

Figure 2.3

For the purposes of this papers, it is convenient to state Theorems 4 and 6 of [3] and Theorem 5 of [2] as follows :

Theorem 2.1 ([3, Theor.4], [2,Theors 1,4]) With the notations of Fig. 23 and the above erminalogy we have :

(i)

$$
\left|m(Q)-h_{12}\left(\log r_{1}+\log r_{2}\right)\right| \leq 0.381 \mathrm{e}^{-\pi h_{12}}
$$

provided that $h_{12}:=h_{1}+h$

(ii)

$$
-\frac{1}{2} \times 0.381 e^{-2 \pi h}{ }_{j} \leq m\left(Q_{j}\right)-h_{j}-\frac{1}{\pi} \log r_{j} \leq 0, j=1,2 .
$$

provided that $h_{j} \geq 1$. 
Theorem 2.2 ([3, Theor. 6], [2, Theor.2]) For the decomposition illustrated in Fig. 23 we have

$$
0 \leq m(Q)-\left\{m\left(Q_{1}\right)+m\left(Q_{2}\right)\right\} \leq 0.761 e^{-2 \pi h},
$$

provided that $h:=\min \left(h_{1}, h_{2}\right) \geq 1$.

Theorem 23 ([2, Theor. 5]) If in Fig. 2.3 the arc $\gamma_{1}$, is a straight line parallel to the real axis, ie. if

$$
\gamma_{1}:=\left\{(x, y): 0 \leq x \leq 1, y=c_{1}<c\right\},
$$

as illustrated in Fig. 2.4, then

$$
0 \leq m(Q)-\left\{h_{1}+m\left(Q_{2}\right)\right\} \leq \frac{1}{2} x 0.381 e^{-2 \pi h_{2}},
$$

provided that $h_{2} \geq 1$.

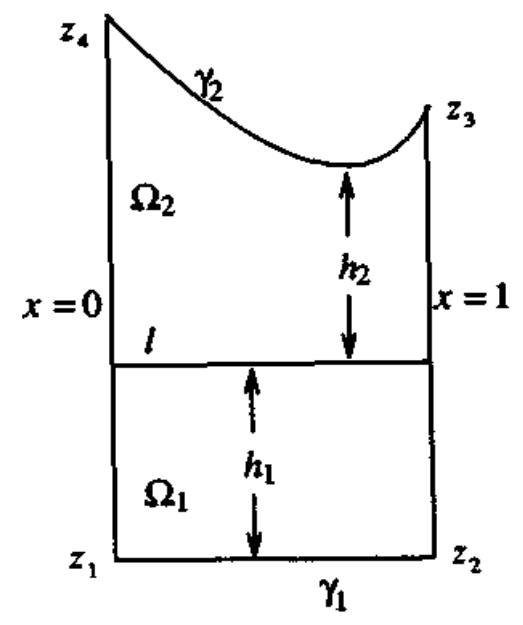

Figure 2.4

We end this section by making the following four remarks:

Remark 2.1 The bounds in (2.1) - (2.4) were deduced from the precise estimates given by Gaier and Hayman in their second paper [3]. For this reason, they are smaller than those given in Theorems 1,4, 2 and 5 of their earlier paper [2].

Remark 2.2 For a general decomposition of the form illustrated in Fig. 2.1 we always have

$$
m(Q) \geq m\left(Q_{1}\right)+m\left(Q_{2}\right)+m\left(Q_{3}\right)
$$

and equality occurs only in the special case where the crosscuts $\gamma_{1}$ and $\gamma_{2}$ are equipotentials of the function $u$ satisfying the Laplacian problem 


$$
\begin{gathered}
-7- \\
\Delta u=0 \text { in } \Omega \\
u=0 \text { on }\left(\mathrm{z}_{1}, \mathrm{z}_{2}\right), \quad u=1 \text { on }\left(\mathrm{z}_{3}, \mathrm{z}_{4}\right), \\
\frac{\partial u}{\partial n}=0 \text { on }\left(z_{2}, z_{3}\right) \cup\left(z_{4}, z_{1}\right),
\end{gathered}
$$

(see e.g. [6, p.437]). In particular, for the special decomposition of Fig. 2.3,

$$
m(Q) \geq m\left(Q_{1}\right)+m\left(Q_{2}\right)
$$

and equality occurs only in the two trivial cases where: (a) $\Omega$ is a rectangle, and (b) $\gamma_{2}$ is the reflection of $\gamma_{1}$ in the crosscut $l$. In addition, for the quadrilaterals $Q, Q_{1}$ and $Q_{2}$ of Fig. 2.3 we have

$$
m(Q)-1 \leq h_{12}:=h_{1}+h_{2} \leq m(Q)
$$

and

$$
m\left(Q_{j}\right)-\frac{1}{2} \leq h_{j} \leq m\left(Q_{j}\right), j=1,2,
$$

(cf. [5, p.36]). Finally, for the quadrilaterals $Q$ and $Q_{2}$ of Fig. 2.4 the composition law (2.6)implies that

$$
m(Q)-\left(h_{1}+h_{2}\right) \geq m\left(Q_{2}\right)-h_{2} .
$$

Remark 23 With reference to the quadrilaterals of Figs 2.3 and 2.4, estimates for the errors in the DDM approximations to $m(Q)$ (similar to those of Theorems 2.2 and 2.3 ), and also for various other errors associated with the DDM approximation to the full conformal map $\mathrm{R}_{m(Q)} \rightarrow \Omega$, were first given by us in [9], without imposing any restrictions on the sizes of $h$ and $h_{2}$. However, the estimates given in [9] were derived by imposing certain additional assumptions on the arcs $\gamma_{1}, \gamma_{2}$, and one of these is rather restrictive. (It requires that the slopes of the two two arcs are numerically less than 1.)

Remark 2.4 Let $\mathrm{T}_{1}$ denote the quadrilateral illustrated in Fig. 2.5. That is, $T_{l}:=\left\{\Omega ; \mathrm{z}_{1}, \mathrm{z}_{2}, \mathrm{z}_{3}, \mathrm{z}_{4}\right\}$ where : (a) $\Omega$ is the trapezium bounded by the real and imaginary axes and the lines $x=1$ and $\mathrm{y}=x+1-1$, with $l>1$, and (b) the points $\mathrm{z}_{1}, \mathrm{z}_{2}, \mathrm{z}_{3}, \mathrm{z}_{4}$ are the corners of $\Omega$.

We note the following, for future reference:

(i) The exact value of $\mathrm{m}\left(T_{l}\right)$ is known exactly, for any $l>1$, in terms of elliptic integrals ( cf. [1, p. 104] ). For example, the exact values of $m\left(T_{2}\right), m\left(T_{3}\right), m\left(T_{3}\right)$ and $m\left(T_{4}\right)$ are given to twelve decimal places by

$$
\begin{aligned}
& m\left(T_{2}\right)=1.279261571171, \quad m\left(T_{3}\right)=2.279364207968, \\
& m\left(T_{3.5}\right)=2.779364391556, \quad m\left(T_{4}\right)=3.279364399489 .
\end{aligned}
$$


(ii) For any $c>0$, Theorem 2.3 gives

$$
0 \leq m\left(T_{l+c}\right)-\left\{m\left(T_{l}\right)+c\right\} \leq \frac{1}{2} \times .0 .381 e^{-2 \pi(l-1)}
$$

Provided that $l \geq 2$.This means, in particular, that

$$
0 \leq m\left(T_{3+c}\right)-\left\{m\left(T_{3}\right)+c\right\} \leq 6.65 \times 10^{-7}
$$

and

$$
0 \leq m\left(T_{4+c}\right)-\left\{m\left(T_{4}\right)+c\right\} \leq 1.25 \times 10^{-9}
$$

It follows that, for any $c>0, m\left({ }^{T_{3+c}}\right)$ can be computed correct to at least five decimal places (from the value of $\mathrm{m}\left(T_{3}\right)$ given in $(2.10 \mathrm{a})$ ), by means of

$$
m\left(T_{3+c}\right)=m\left(T_{3}\right)+c
$$

Similarly, $m\left({ }^{T_{4+c}}\right)$ can be computed correct to at least eight decimal places (from the value of $m\left(T_{4}\right)$ given in (2.10b)) by means of

$$
m\left(T_{4+c}\right)-m\left(T_{4}\right)+c
$$

( see also [8, pp. 78-81] and [10, Ex. 3.1]).

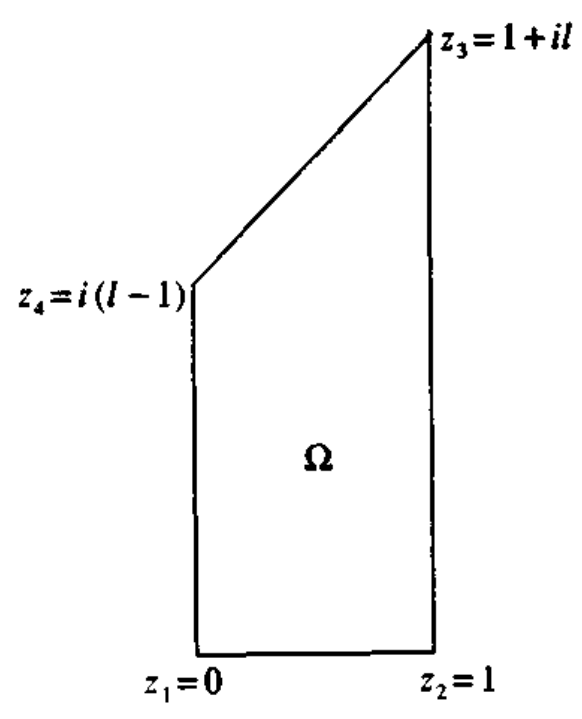

Figure 2.5

\section{Two theorems for general domain decomposition}

In this section we prove two theorems concerning general domain decompositions of the form illustrated in Fig. 3.1(a). As will become apparent, these two theorems are simple consequences of the results of Gaier and Hayman stated in Section 2. 


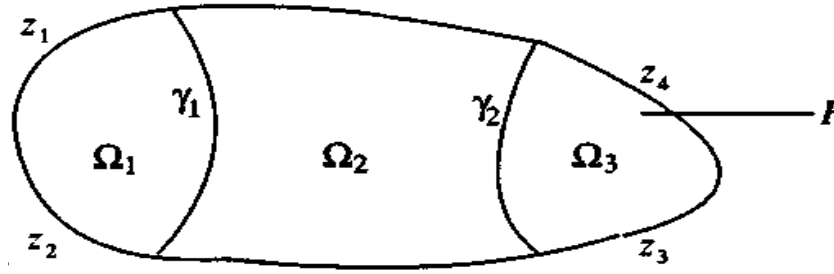

(a)

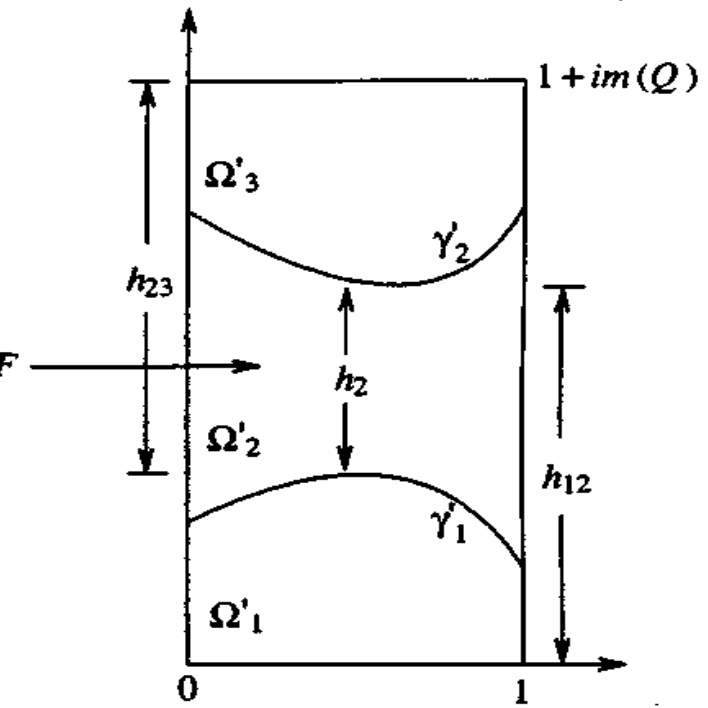

(b)

Figure 3.1

Theorem 3.1 With the notations of Fig. 3.1(a) and the terminology introduced in Section 2, we have

$$
\left|m(Q)-\left\{m\left(Q_{1,2}\right)+m\left(Q_{2,3}\right)-m\left(Q_{2}\right)\right\}\right| \leq 8.82 e^{-\pi m\left(Q_{2}\right)}
$$

provided that $m\left(Q_{2}\right) \geq 3$.

Proof The quadrilateral $Q:=\left\{\Omega ; \mathrm{z}_{1}, \mathrm{z}_{2}, \mathrm{z}_{3}, \mathrm{z}_{4}\right\}$ is conformally equivalent to the rectangular quadrilateral

$$
Q^{\prime}:=\left\{R_{m(Q)} ; 0,1,1+i m(Q), i m(Q)\right\}
$$

where

$$
R_{m(Q)}:=\{(\xi, \eta) ; 0<\xi<1,0<\eta<m(Q)\}
$$

Let $F$ be the corresponding conformal map of $Q$ onto $Q^{\prime}$, and let $\gamma_{1}^{\prime}, \gamma_{2}^{\prime}$ be respectively the images of the crosscuts $\gamma_{1}, \gamma_{2}$ under this map. Then, $\gamma_{1}^{\prime}$ and $\gamma_{2}^{\prime}$ define the decomposition of $Q^{\prime}$ illustrated in Fig. 3.1(b), where

$$
\left.m\left(Q_{j}^{\prime}\right)=m\left(Q_{j}\right), j=1,2,3, \quad m\left(Q_{1.2}^{\prime}\right)=m\left(Q_{1,2}\right) \text { and } m^{\left(Q_{2,3}^{\prime}\right.}\right)=m\left(Q_{2,3},\right),
$$

because the modules are conformally invariant.

Let

$$
h_{2}:=\min \left\{\operatorname{Im} z^{\prime}: z^{\prime} \in \gamma_{2}^{\prime}\right\}-\max \left\{\operatorname{Im} z^{\prime}: z^{\prime} \in \gamma_{1}^{\prime}\right\}
$$




$$
h_{12}:=\min \left\{\operatorname{Im} z^{\prime} ; z^{\prime} \in \gamma_{2}^{\prime}\right\}, h_{23}:=m(Q)-\max \left\{\operatorname{Im} z^{\prime} ; z^{\prime} \in \gamma_{1}^{\prime}\right\}
$$

and observe that

$$
h_{12}+h_{23}-h_{2}=m(Q) \text {, }
$$

Observe also that the assumption $m\left(Q_{2}\right) \geq 3$ implies the fol lowing :

$$
\begin{gathered}
h_{2} \geq m\left(Q_{2}\right)-1 \geq 2, \\
h_{12} \geq m\left(Q_{1,2}\right)-\frac{1}{2} \geq m\left(Q_{2}\right)-\frac{1}{2} \geq \frac{5}{2} \\
h_{23} \geq m\left(Q_{2,3}\right)-\frac{1}{2} \geq m\left(Q_{2}\right)-\frac{1}{2} \geq \frac{5}{2}
\end{gathered}
$$

\section{(see Remark 2.2 ).}

Next, apply the results of Theorem 2.1 to each of the quadrilaterals $Q_{2}^{\prime}, Q_{1,2}^{\prime}$ and $Q_{2,3}^{\prime}$. This, in conjunction with (3.2), gives

$$
\begin{aligned}
& \mid m\left(Q_{2}\right)-h_{2}-\frac{1}{\pi}\left(\log r_{1}+\log r_{2} \mid \leq 0.381 e^{-\pi h_{2}}\right. \\
& -\frac{1}{2} \times 0.381 \mathrm{e}^{-2 \pi \mathrm{h}_{12}} \leq \mathrm{m}\left(\mathrm{Q}_{1,2}\right)-\mathrm{h}_{12}-\frac{1}{\pi} \log \mathrm{r}_{2} \leq 0, \\
& -\frac{1}{2} \times 0.381 \mathrm{e}^{-2 \pi \mathrm{h}_{23}} \leq \mathrm{m}\left(\mathrm{Q}_{2,3}\right)-\mathrm{h}_{23}-\frac{1}{\pi} \log \mathrm{r}_{1} \leq 0,
\end{aligned}
$$

where $r_{1}$ and $\mathrm{r}_{2}$ are respectively the exponential radii of the arcs $\gamma_{1}^{\prime}$ and. $\overline{\gamma_{2}^{\prime}}$ It follows that

$$
-E_{1} \leq m(Q)-\left\{m\left(Q_{1,2}\right)+m\left(Q_{2,3}\right)-m\left(Q_{2}\right)\right\} \leq E_{2},
$$

where, by using (3.4)-(3.6),

$$
E_{1}:=0.381 e^{-\pi h_{2}} \leq 0.381 e^{\pi} e^{-\pi m\left(Q_{2}\right)} \leq 8.82 e^{-\pi m\left(Q_{2}\right)}
$$

and

$$
E_{2}:=0.381\left(e^{-\pi h_{2}}+\frac{1}{2} e^{-2 \pi h_{12}}+\frac{1}{2} e^{-2 \pi h}\right) \leq 8.82 e^{-\pi n\left(Q_{2}\right)}
$$

This completes the proof of the theorem.

Theorem 3.2 Consider the decomposition illustrated in Fig. 3.1(a) and, with the terminology used in the proof of Theorem 3.1, assume that the image of the crosscut $\gamma_{1}$ under the conformal map $F: Q \rightarrow Q^{\prime}$ is a straight line parallel to the real axis (see Fig. 3.2 ). Then,

$$
-4.41 \mathrm{e}^{-2 \pi \mathrm{m}\left(\mathrm{Q}_{2}\right)} \leq \mathrm{m}(\mathrm{Q})-\left\{\mathrm{m}\left(\mathrm{Q}_{1,2}\right)+\mathrm{m}\left(\mathrm{Q}_{2,3}\right)-\mathrm{m}\left(\mathrm{Q}_{2}\right)\right\} \leq 0,
$$

provided that $m\left(Q_{2}\right) \geq 1.5$.

Proof The assumption $m\left(Q_{2}\right) \geq 1.5$ implies that

$$
h_{2} \geq m\left(Q_{2}\right)-\frac{1}{2} \geq 1
$$


(see (2.8)). Therefore, for the quadrilateral $Q_{1,2}^{\prime}$ of Fig. 3.2, Theorem 2.3 gives

$$
0 \leq m\left(Q_{1,2}\right)-\left\{h_{1}+m\left(Q_{2}\right)\right\} \leq E
$$

where, by using (3.8),

$$
\mathrm{E}:=\frac{1}{2} \times 0.381 \mathrm{e}^{-2 \pi \mathrm{L}_{2}} \leq \frac{1}{2} \times 0.381 \mathrm{e}^{\pi} \mathrm{e}^{-2 \pi \mathrm{m}\left(\mathrm{Q}_{2}\right)} \leq 4.41 \mathrm{e}^{-2 \pi \mathrm{m}\left(\mathrm{Q}_{2}\right)}
$$

The desired result follows at once from (3.9), because

$$
h_{1}=m(Q)-h_{23}=m(Q)-m\left(Q_{2.3}\right) \text {. }
$$

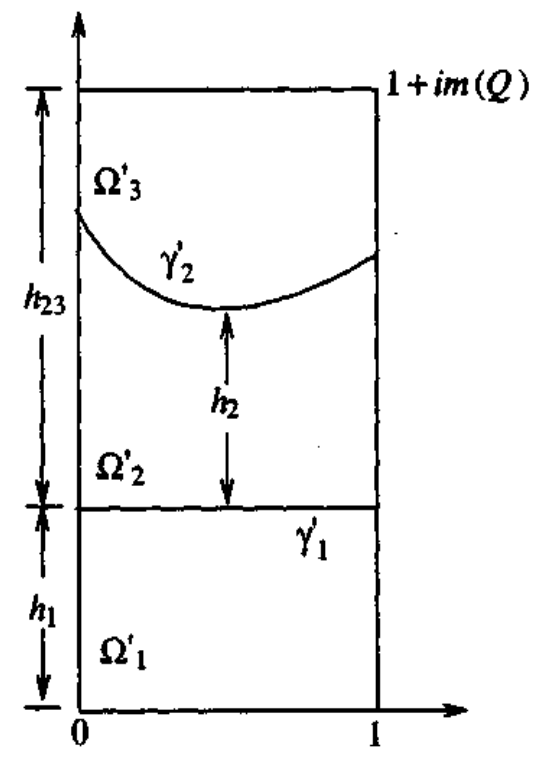

Figure 3.2

\section{Applications}

4.1 Let $Q_{1}$ and $Q_{2}$ be the two component quadrilaterals of the decomposition illustrated in Fig. 4.1(a), and assume that the reflection $\hat{\gamma}_{1}$ of the arc $\gamma_{1}$, in the crosscut

lies above the $\operatorname{arc} \gamma_{2}$. Then,

$$
l:=\{(x, y): 0 \leq x \leq 1, y=x\}
$$

$$
0 \leq m(Q)-\left\{m\left(Q_{1}\right)+m\left(Q_{2}\right)\right\} \leq 4.41 e^{-2 \pi m\left(Q_{2}\right)}
$$

provided that $m\left(Q_{2}\right) \geq 1.5$. This can be shown as follows :

Reflect $\Omega_{1}$, in $l$, and consider the decomposition of the resulting quadrilateral $\hat{Q}$ illustrated in Fig. 4.1(b). (That is the decomposition of $\hat{Q}$ is defined by the two crosscuts $l$ and $\gamma_{2}$.) Then, because of the symmetry, 


$$
m\left(Q_{2,3}\right)=m\left(Q_{1}\right), \quad m(\hat{Q})=2 m\left(Q_{1}\right)
$$

and the image of the crosscut $l$ under the associated conformal map

will be the straight line

$$
F: \hat{Q} \rightarrow\left\{R_{m(\hat{Q})} ; 0,1,1+i m(\hat{Q}), i m(\hat{Q})\right\}
$$

$$
\left\{(\xi, \eta) ; 0 \leq \xi \leq 1, \eta=m\left(Q_{1}\right)\right\}
$$

Therefore, Theorem 3.2 is applicable to the decomposition of Fig. 4.1(b), and its application gives

$$
-4.41 e^{-2 \pi m\left(Q_{2}\right)} \leq m(\hat{Q})-\left\{m\left(Q_{1,2}\right)+m\left(Q_{2.3}\right)-m\left(Q_{2}\right)\right\} \leq 0
$$

Since $m\left(Q_{12}\right),=m(Q)$ the, desired result follows at once from (4.2) and (4.3).

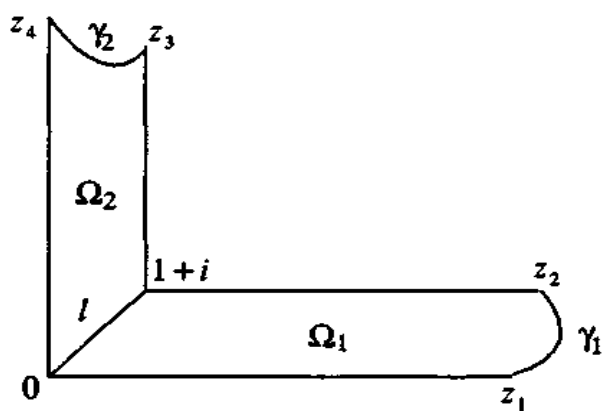

(a)

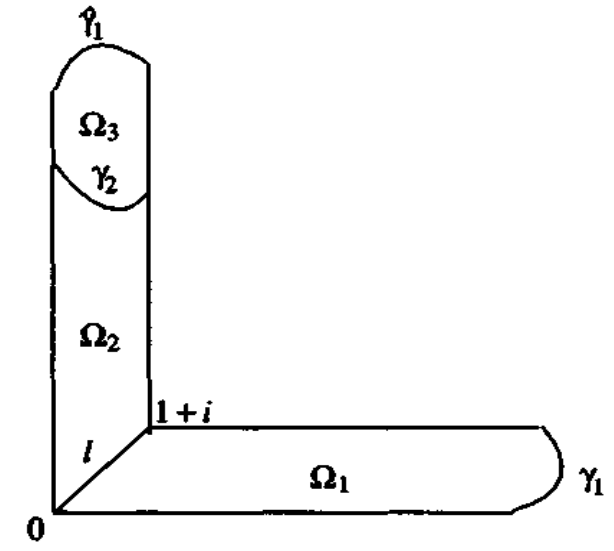

(b)

Figure 4.1

4.2 Let $Q_{1}, Q_{2}$, and $Q_{3}$ be the three component quadrilaterals of the decomposition illustrated in Fig. 4.2, let $\hat{\Omega}_{1}$, and $\hat{\Omega}_{3}$ denote thereflections of $\Omega_{1}$, and $\Omega_{3}$ in the crosscuts $l_{1}$, and $l_{2}$, and assume that $\Omega_{2} \subset \hat{\Omega}_{1}$ and $\Omega_{2} \subset \hat{\Omega}_{3}$. Then,

$$
0 \leq m(Q)-\left\{m\left(Q_{1}\right)+m\left(Q_{2}\right)+m\left(Q_{3}\right)\right\} \leq 8.821 e^{-\pi m\left(Q_{2}\right)}
$$

provided that $m\left(Q_{2}\right) \geq 3$. This can be shown as follows:

The application of Theorem 3.1 gives

$$
\left|m(Q)-\left\{m\left(Q_{1,2}\right)+m\left(Q_{2,3}\right)+m\left(Q_{2}\right)\right\}\right| \leq 8.82 e^{-\pi m\left(Q_{2}\right)}
$$

Further, the application of estimate (4.1) to each of the quadrilaterals $Q_{1,2}$ and $Q_{2,3}$ gives 


$$
0 \leq m\left(Q_{1,2}\right)-\left\{m\left(Q_{1}\right)+m\left(Q_{2}\right)\right\} \leq 4.41 e^{-2 \pi n\left(Q_{2}\right)}
$$

and

$$
0 \leq m\left(Q_{2,3}\right)-\left\{m\left(Q_{2}\right)+m\left(Q_{3}\right)\right\} \leq 4.41 e^{-2 \pi m\left(Q_{2}\right)}
$$

The desired result then follows at once from (4.5)-(4.7) and the composition law (2.5).

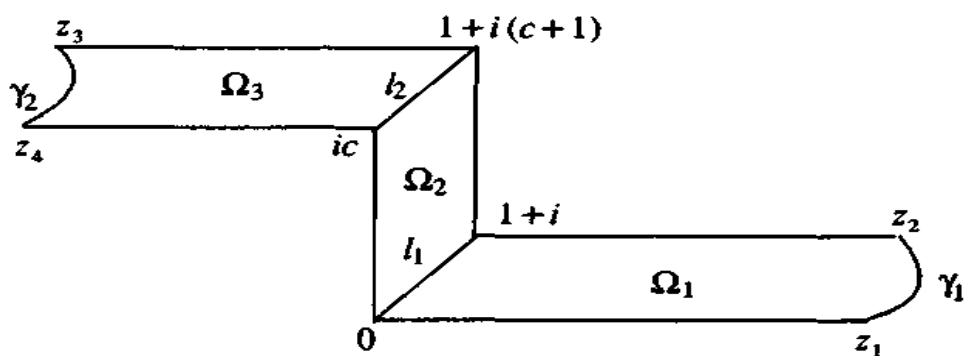

Figure 4.2

4.3 Consider the decomposition illustrated in Fig.4.3, let $\hat{\Omega}_{1}$ and $\hat{\Omega}_{3}$ denote respectively the reflections of $\Omega_{1}$ and $\Omega_{3}$ in the crosscuts $l_{1}$, and $l_{2}$, and assume that $\Omega_{2} \subset \hat{\Omega}_{1}$ and. $\Omega_{2} \subset \hat{\Omega}_{3}$ Then, an argument similar to that used in $\S 4.2$ above gives

$$
0 \leq m(Q)-\left\{m\left(Q_{1}\right)+m\left(Q_{2}\right)+m\left(Q_{3}\right)\right\} \leq 8.821 \mathrm{e}^{-\pi m\left(Q_{2}\right)}
$$

provided that $m\left(Q_{2}\right) \geq 3$.

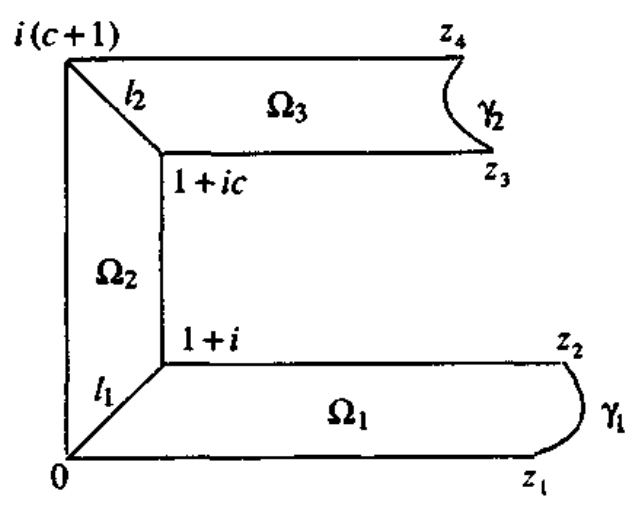

Figure 43

4.4 Consider the decomposition of the quadrilateral $Q:=\left\{\Omega ; \mathrm{z}_{1}, \mathrm{z}_{2}, \mathrm{Z}_{3}, \mathrm{z}_{4}\right\}$ illustrated in Fig.4.4(a), where: 
(i) The domain $\Omega$ is the union of a rectangle

$$
\{(x, \mathrm{y}): 0<x<2 c, 0<\mathrm{y}<1\}
$$

and some other simply-connected domain,

(ii) The decomposition is defined by the straight line crosscut

$$
l:=\{(x, \mathrm{y}): x=c, 0 \leq \mathrm{y} \leq 1\}
$$

ie. $m\left(Q_{2}\right)=c$.

Then,

$$
0 \leq m(Q)-\left\{c+m\left(Q_{1}\right\} \leq 4.41 e^{-2 \pi c},\right.
$$

provided that $\mathrm{c} \geq 1.5$.

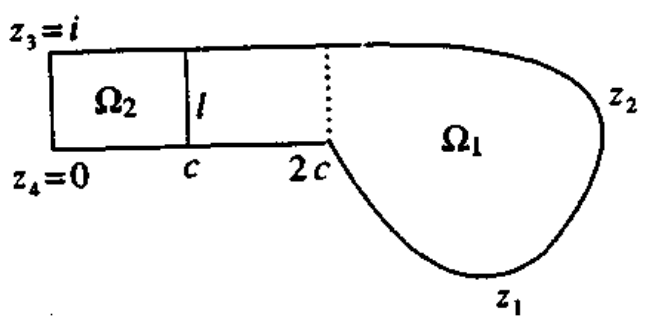

(a)

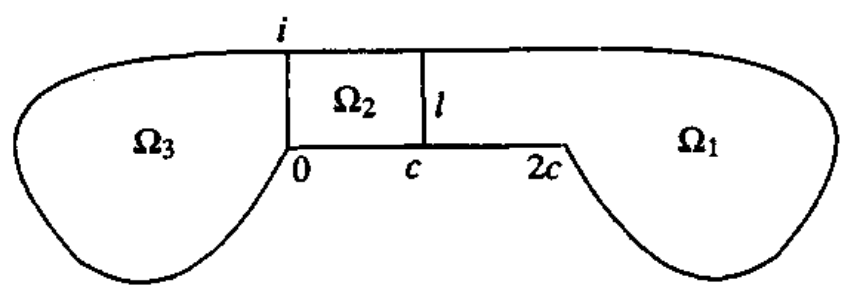

Figure 4.4

The estimate (4.11) can be derived, in a manner similar to that used in $\S 4.1$, by reflecting the subdomain $\Omega_{1}$, in the crosscut $l$ and considering the decomposition of the resulting quadrilateral $\hat{Q}$ illustrated in Fig. 4.4(b). Then, because $l$ is a line of symmetry, the application of Theorem 3.2 to this decomposition of $\hat{Q}$ gives

$$
-4.41 e^{-2 \pi m\left(Q_{2}\right)} \leq m(\hat{Q})-\left\{m\left(Q_{1.2}\right)+m\left(Q_{2,3}\right)-m\left(Q_{2}\right)\right\} \leq 0
$$


The desired result follows at once from (4.12), because

$$
m(\hat{Q})=2 m\left(. Q_{l}\right), m\left(Q_{2}\right)=c, m\left(Q_{1,2}\right)=m(Q) \text { and } m\left(Q_{2,3}\right)=m\left(Q_{l}\right) .
$$

4.5 Let $Q_{1}$ and $Q_{2}$ be the component quadrilaterals of the decomposition illustrated in Fig. 4.5(a), where again the domain $\Omega$ consists in part by a rectangle of the form (4.9), and the crosscut is the straight line (4.10). Then,

$$
0 \leq m(Q)-\left\{m\left(Q_{1}\right)+m\left(Q_{2}\right)\right\} \leq 17.64 e^{-2 \pi c}
$$

provided that $c \geq 1.5$.

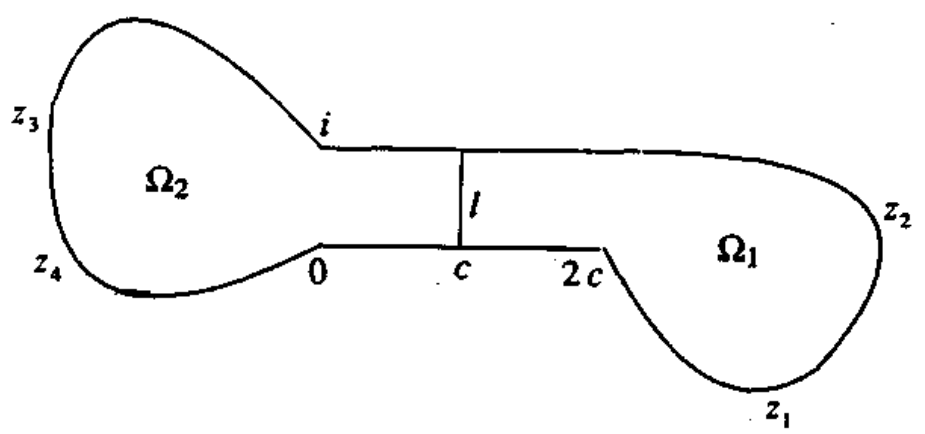

(a)

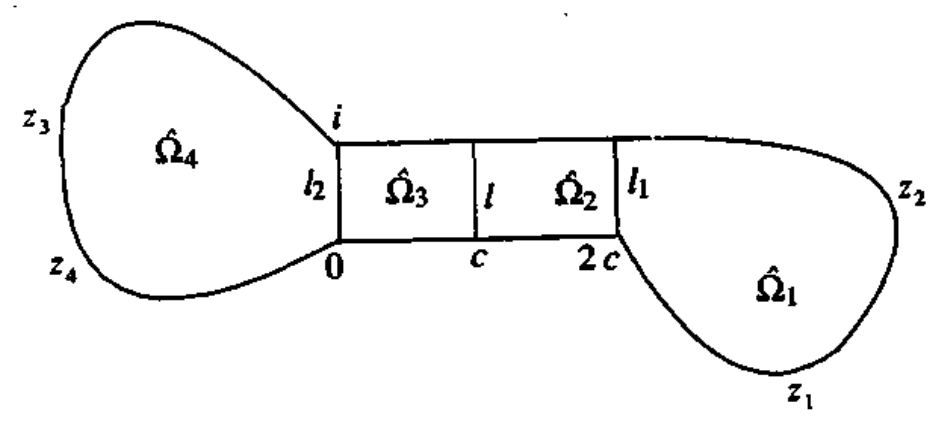

(b)

Figure 4.5

For the proof of (4.13), we introduce two additional crosscuts by means of the straight lines

$$
l_{1}:=\{(x, y): x=2 \mathrm{c}, 0 \leq y \leq 1\} \text { and } l_{2}:=\{(x, y): x=0,0 \leq y \leq 1\} \text {, }
$$

and label the subdomains of the resulting decomposition $\hat{\Omega}_{1}, \hat{\Omega}_{2}, \hat{\Omega}_{3}, \hat{\Omega}_{4}$ as illustrated inFig.4.5(b). Then, the application of Theorem 3.1 to the decomposition of $Q$ defined by the two crosscuts $l_{1}$ and $l_{2}$ gives

$$
\left|m(Q)-\left\{m\left(\hat{Q}_{1,2,3}\right)+m\left(\hat{Q}_{2,3,4}\right)-2 c\right\}\right| \leq 8.82 e^{-2 \pi c},
$$

because $m\left(\hat{Q}_{2,3}\right)=2$ c. In addition, the application of (4.11) to each of the quadrilaterals $\hat{Q}_{1,2,3}$ and 
$Q_{2,3,4}$, gives

$$
0 \leq m\left(\hat{Q}_{1,2,3}\right)-\left\{c+m\left(\hat{Q}_{1,2,}\right)\right\} \leq 4.41 e^{-2 \pi c}
$$

and

$$
0 \leq m\left(\hat{Q}_{2,3,4}\right)-\left\{c+m\left(\hat{Q}_{3,4}\right)\right\} \leq 4.41 e^{-2 \pi c}
$$

The desired result follows from (4.14)-(4.16), by observing that $m\left(\hat{Q}_{1,2}\right)=m\left(Q_{1}\right)$ and $m\left(\hat{Q}_{3,4}\right)=m\left(Q_{2}\right)$.

\section{Numerical examples}

Example 5.1 Let $Q:=\left\{\Omega ; \mathrm{z}_{1}, \mathrm{z}_{2}, \mathrm{z}_{3}, \mathrm{z}_{4}\right\}$ be the quadrilateral illustrated in Fig. 5.1, where the two boundary $\operatorname{arcs} \gamma_{1}, \gamma_{2}$, have cartesian equations

$$
\begin{gathered}
x=0.25 y^{4}-0.5 y^{2}+7,0 \leq y \leq 1, \\
x=7.5+0.25 \cos 2 \pi(6-y), \quad 6 \leq y \leq 7,
\end{gathered}
$$

respectively.

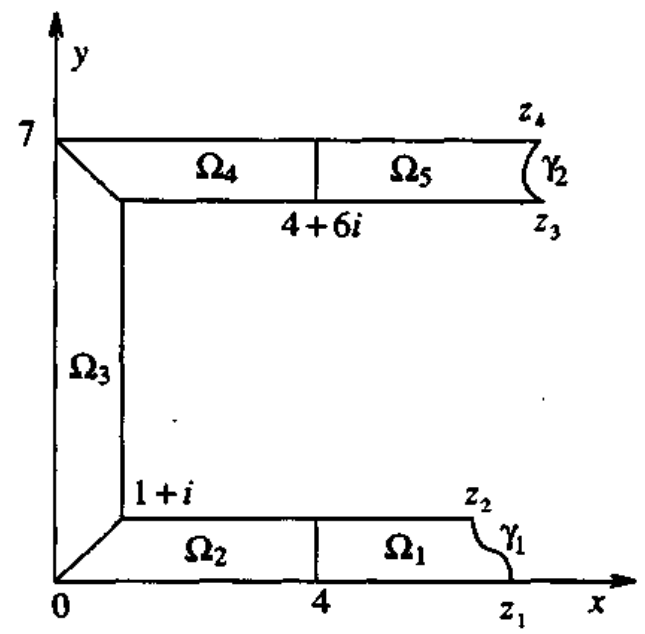

Figure 5.1

We consider approximating $m(Q)$ by

$$
m Q=\sum_{\mathrm{j}=1} m Q_{j}
$$

and note the following in connection with the determination of the modules of the component quadriclaterals $Q j, j=1, \ldots . .5$ : 
(i) The modules of quadrilaterals of the form illustrated in Fig. 2.3 can be determined easily by means of the Garrick iterative algorithm given in [4]. In addition, such quadrilaterals admit further decompositions to which the estimates (2.3) and (2.4) of Gaier and Hayman are applicable. For the component quadrilaterals $Q_{1}$, and $Q_{5}$ under consideration the algorithm of [4] gives the values

$$
m\left(Q_{1}\right)=2.859569035 \text { and } m\left(Q_{5}\right)=3.364089632,
$$

and these are expected to be correct to all the figures quoted (cf. [10, Ex. 3.2]).

(ii) As was indicated in $\S 2$, the values of $m\left(Q_{2}\right), m\left(Q_{3}\right)$ and $m\left(Q_{4}\right)$ are known exactly in terms of elliptic integrals. More specifically, if $T_{l}$ denotes the quadrilateral of Fig. 2.5, then these three modules are given correct to nine decimal places by

and

$$
m\left(Q_{2}\right)=m\left(Q_{4}\right)=m\left(T_{4}\right)=3.279364399
$$

$$
m\left(Q_{3}\right)=2 m\left(T_{3.5}\right)=5.558728783
$$

(cf. Remark 2.4 ).

Thus, $\widetilde{m}(Q)$ is given by

$$
\tilde{m}(Q)=18.341116249 \text {. }
$$

For the error in the above approximation, we first note that estimate (4.8) implies

$$
0 \leq m(Q)-\left\{m\left(Q_{1,2}\right)+m\left(Q_{3}\right)+m\left(Q_{4,5}\right)\right\} \leq 8.821 e^{-\pi m\left(Q_{3}\right)},
$$

(cf. $§ 4.3$ ). In addition, Theorem 2.2 implies

$$
0 \leq m\left(Q_{1,2}\right)-\left\{m\left(\mathrm{Q}_{1}\right)+m\left(Q_{2}\right)\right\} \leq 0.761 \text {, and } e^{-2 \pi h_{1}},
$$

and

$$
0 \leq \mathrm{m}\left(\mathrm{Q}_{4,5}\right)-\left\{\mathrm{m}\left(\mathrm{Q}_{4}\right)+\mathrm{m}\left(\mathrm{Q}_{5}\right)\right\} \leq 0.761 e^{-2 \pi h_{4}},
$$

where $h_{1}=2.75$ and $h_{4}=3$. Therefore,

where

$$
0 \leq m(Q))^{\sum_{j=1}^{5} m\left(Q_{j}\right) \leq E},
$$

$$
\mathrm{E}:=8.821 e^{-\pi m\left(Q_{3}\right)}+0.761\left(e^{-2 \pi h_{1}}+e^{-2 \pi h_{4}}\right) \leq 2.6 \times 10^{-7},
$$

ie.

$$
0 \leq m(Q)-\tilde{m}(Q) \leq 2.6 \times 10^{-7}
$$


Thus,

Example 5.2 Let $Q:=\left\{\Omega ; \mathrm{z}_{1}, \mathrm{z}_{2}, \mathrm{z}_{3}, \mathrm{z}_{4}\right\}$ be the quadrilateral illustrated in Fig 5.2, where the boundary arcs $\gamma_{1}, \gamma_{2}$, have cartesian equations

$$
\begin{gathered}
x=y(y-1)+6.5, \quad 0 \leq y \leq 1, \\
x=-7.5-0.2 \operatorname{sech}^{2} 2.5 y, 0 \leq y \leq 1,
\end{gathered}
$$

respectively.

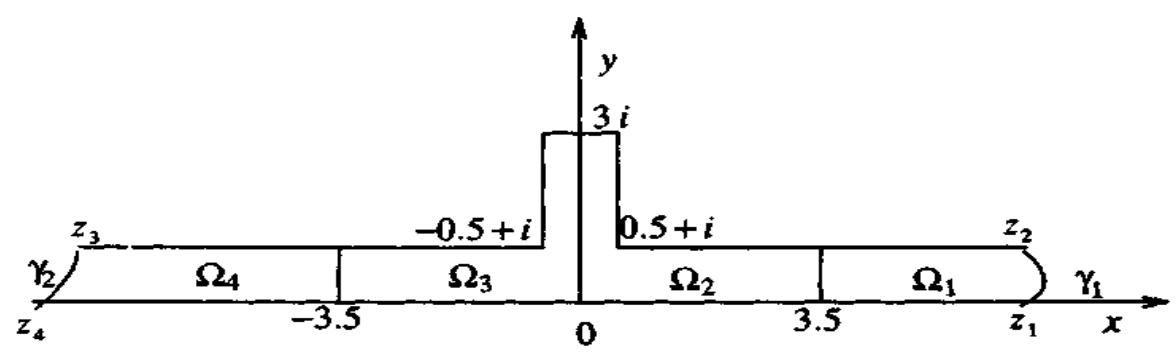

Figure 5.2

Here we approximate $m(Q)$ by

$$
\tilde{m}(Q):=\sum_{\mathrm{j}=1}^{5} \mathrm{~m}\left(\mathrm{Q}_{\mathrm{j}}\right),
$$

where:

(i) The modules of $Q_{1}$ and $Q_{4}$ are determined by using the Garrick algorithm of [4]. The computed values are

$$
m\left(Q_{1}\right)=3.12181333 \text { and } m\left(Q_{4}\right)=4.06551571 \text {, }
$$

and these are expected to be correct to all the figures quoted (cf. [10, Ex.3.3]).

(ii) The module of $Q_{2}$ (which, by symmetry, is equal to that of $Q_{3}$ ) is determined by using the subroutine RESIST of the Schwarz-Christoffel package SCPACK of Terefethen [11]. This gives the value

$$
m\left(Q_{2}\right)=3.42344546=m\left(Q_{3}\right),
$$

which is also expected to be correct to all the figures quoted. 
Thus, $\widetilde{m}(Q)$ is given by

$$
\tilde{m}(Q):=m\left(Q_{1}\right)+2 m\left(Q_{2}\right)+m\left(Q_{4}\right)=14.03421996 .
$$

Forthe error in the aboveDDMapproximation, we first apply estimate (4.13), of $\S 4.5$, to the decomposition of $Q$ defined by the single crosscut at $\mathrm{x}=3.5$. This gives

$$
0 \leq m(Q)-\left\{m\left(Q_{1}\right)+m\left(Q_{2,3,4}\right)\right\} \leq 17.64 e^{-6 \pi} .
$$

The same estimate, when applied to the decomposition of $Q_{2,3,4}$ defined by the crosscut at $x=-3.5$, gives

where, by symmetry,

$$
0 \leq m\left(Q_{2,3,4}\right)-\left\{m\left(Q_{2,3}\right)+m\left(Q_{4}\right)\right\} \leq 17.64 e^{-6 \pi},
$$

$$
m\left(Q_{2,3}\right)=m\left(Q_{2}\right)+m\left(Q_{3}\right)=2 m\left(Q_{2}\right)
$$

Therefore, from (5.1) - (5.3), we have that

ie.

$$
0 \leq m(Q)-\tilde{m}(Q) \leq 35.28^{e^{-6 \pi}} \leq 2.3 \times 10^{-7}
$$

$$
14.03421996 \leq \mathrm{m}(Q) \leq 14.03422019 \text {. }
$$

Example 5.3 Let $Q:=\left\{\Omega ; \mathrm{z}_{1}, \mathrm{z}_{2}, \mathrm{z}_{3}, \mathrm{z}_{4}\right\}$ be the quadrilateral illustrated in Fig. 5.3, where the width of each strip of the spiral $\Omega$ is 1 , and the lengths of the "outer" segments of $\partial \Omega$ (in clockwise

order, starting from the right hand side) are 18,19,18,16,15,13,12,10,9,7,6,4 and 3 .

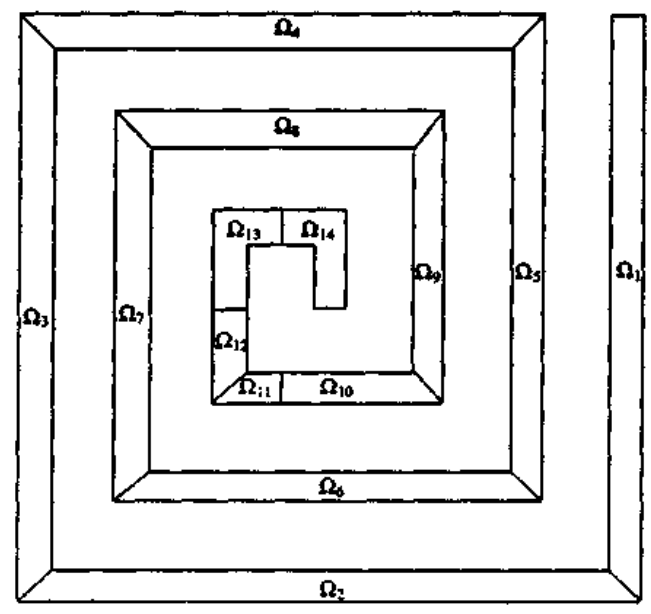

Figure 5.3 
This is one of the examples considered by Howell and Trefethen [7], in connection with the use of a modified Schwarz Christoffel technique for the conformal mapping of elongated polygonal regions. Here we consider approximating $m(Q)$ by

$$
\tilde{m}(Q):=\sum_{j=1}^{9} m\left(Q_{j}\right)+m\left(Q_{10,11}\right)+\sum_{j=12}^{14} m\left(Q_{j}\right),
$$

and note the following concerning the decomposition used and the determination of the corresponding component quadrilaterals:

(i) The crosscut that subdivides $Q_{10,11}$ into $Q_{10}$ and $Q_{11}$ is needed only for estimating the error in he DDM approximation (5.4).

(ii) Let $T_{1}$, be the quadrilateral of Fig. 2.5. Then,

$$
\begin{gathered}
m\left(Q_{1}\right)=m\left(T_{18}\right), \quad m\left(Q_{2}\right)=2 m\left(T_{9.5}\right), \quad m\left(Q_{3}\right)=2 m\left(T_{9}\right), \\
m\left(Q_{4}\right)=2 m\left(T_{8}\right), \quad m\left(Q_{5}\right)=2 m\left(T_{7.5}\right), \quad m\left(Q_{6}\right)=2 m\left(T_{6.5}\right), \\
m\left(Q_{7}\right)=2 m\left(T_{6}\right), \quad m\left(Q_{8}\right)=2 m\left(T_{5}\right), \quad m\left(Q_{9}\right)=2 m\left(T_{4.5}\right), \\
m\left(Q_{10,11}\right)=2 m\left(T_{3.5}\right), \text { and } m\left(Q_{12}\right)=m\left(T_{3}\right),
\end{gathered}
$$

This means that all the above modules can be written down, correct to at least eight decimal places, by using the values of $\mathrm{m}\left(T_{3}\right), m\left(T_{3.5}\right)$ and $m\left(T_{4}\right)$, given in (2.10), in conjunction with the formula

$$
m\left(T_{4+c}\right)=m\left(T_{4}\right)+c, c>0,
$$

( cf. Remark 2.4 ).

(iii) By symmetry

$$
m\left(Q_{11,12}\right)=m\left(Q_{13}\right)=m\left(Q_{14}\right)
$$

and

$$
m\left(Q_{11, \ldots, 14}\right)=3 m\left(Q_{13}\right)
$$

(iv) For the computation of $m\left(Q_{13}\right)$ we use (as in Example 5.2 ) the subroutine RESIST of [11]. This gives the value

$$
m\left(Q_{13}\right)=3.558625812
$$

which is expected to be correct to all the figures quoted.

Thus, by determining the modules involved in (5.4), as indicated in (ii) and(iv) above, we find that

$$
\widetilde{m}(Q)=132.70453941 \text {. }
$$


This should be compared with the approximation

$$
m(Q)=132.70454
$$

obtained by Howell and Trefethen [7. p.943].

The error in $\widetilde{m}(Q)$ can be estimated as follows :

Theorem 3.1 implies that

$$
\left|m(Q)-\left\{m\left(\mathrm{Q}_{1,2}\right)+m\left(Q_{2, \ldots, 14}\right)-m(Q 2)\right\}\right| \leq 8.82 e^{-\pi m\left(Q_{2}\right)} .
$$

Also, the application of estimate (4.1) to $Q_{1,2}$ gives

$$
0 \leq m\left(Q_{1,2}\right)-\left\{\mathrm{m}\left(Q_{1}\right)+m\left(Q_{2}\right)\right\} \leq 4.41 \mathrm{e}^{-2 \pi \mathrm{m}\left(\mathrm{Q}_{2}\right)} .
$$

Hence, by recalling (2.5),

$$
0 \leq m(Q)-\left\{\mathrm{m}\left(\mathrm{Q}_{1}\right)+\mathrm{m}\left(\mathrm{Q}_{2, \ldots 14}\right)\right\} \leq 8.82 e^{-\pi n\left(Q_{2}\right)}+4.41 \mathrm{e}^{-2 \pi \pi \mathrm{m}(2)}
$$

Similarly, by combining (5.9) with the results obtained by applying respectively Theorem 3.1 and estimate (4.1) to $Q_{2, \ldots 14}$ and $Q_{2 . .3} ; Q_{3, \ldots 14}$ and $Q_{3,4, \ldots, \ldots ;} Q_{8, \ldots, 14}$ and $Q_{8, \ldots, 14}$ and $Q_{8,9 ;} Q_{9, \ldots, 14}$ and $Q_{9,10,11 ;}$ we find that

$$
0 \leq m(Q)-\left\{\sum_{j=1}^{9} m\left(Q_{j}\right)+m\left(Q_{10, \ldots 14}\right)\right\} \leq E_{1}
$$

where

$$
\mathrm{E}_{1}:=8.82\left\{\sum_{j=2}^{9} e^{-2 \pi n\left(Q_{j}\right)}+e^{-2 \pi m\left(Q_{10,11}\right)}\right\}+4.41\left\{e^{-2 \pi m\left(Q_{1}\right)}+\sum_{\mathrm{j}=3}^{9} e^{-2 \pi n\left(Q_{j}\right)}+e^{-2 \pi n\left(Q_{10,11}\right)}\right\}
$$

Next, by applying Theorem 3.1 to $Q_{10, \ldots 14}$ and estimate (4.1) to $Q_{10,11,12 \text {, we obtain }}$

and

$$
0 \leq m\left(Q_{10, \ldots, 14}\right)-\left\{m\left(Q_{10,11,12}\right)+m\left(Q_{11, \ldots, 14}\right)-m\left(Q_{11,12}\right)\right\} \leq 8.82 e^{-\pi m\left(Q_{11,12}\right)}
$$

$$
0 \leq m\left(Q_{10,11,12}\right)-\left\{m\left(Q_{10,11}\right)+m\left(Q_{12}\right)-m\left(Q_{11,12}\right)\right\} \leq 4.41 \mathrm{e}^{-2 \pi \mathrm{m}\left(\mathrm{Q}_{12}\right)}
$$

These two estimates, in conjunction with the symmetry relations (5.6), then yield

$$
0 \leq \mathrm{m}\left(\mathrm{Q}_{10 \ldots \ldots ., 14}\right)-\left\{\mathrm{m}\left(\mathrm{Q}_{10,11}\right)+\sum_{\mathrm{j}=12}^{14} \mathrm{~m}\left(\mathrm{Q}_{\mathrm{j}}\right) \leq \mathrm{E}_{2},\right.
$$

where

$$
E_{2}:=8.82 e^{-\pi m\left(Q_{13}\right)}+4.41 e^{-2 \pi m\left(Q_{12}\right)} .
$$

Finally, by combining (5.10) and (5.11), we obtain

$$
0 \leq m(Q)-\tilde{m}(Q) \leq E
$$

where

$$
E:=E_{1}+E_{2} \leq 1.26 \times 10^{-4}
$$


Therefore, from (5.7) and (5.12),

$$
132.704539 \leq \mathrm{m}(\mathrm{Q}) \leq 132.704666
$$

We end by considering the alternative decomposition of $Q$ illustrated in Fig. 5.4. In this, the component quadrilaterals $Q_{j}, j=1,2, \ldots 9$, are the same as those in Fig. 5.3, ie. $m\left(Q_{j}\right), j=1,2, \ldots 9$, are given by $(5.5 \mathrm{a})-(5.5 \mathrm{c})$. In addition,

$$
m\left(Q_{10}\right)=2 m\left(T_{3.5}\right), m\left(Q_{11}\right)=2 m\left(T_{3}\right), m\left(Q_{12}\right)=2 m\left(T_{2}\right) \text { and } m\left(Q_{13}\right)=m\left(T_{3}\right) .
$$

Thus, the corresponding DDM approximation $\hat{m}(Q)$ can be calculated trivially (as indicated in (ii) above) from the fourvalues of $m\left(T_{2}\right), m\left(T_{3}\right), m\left(T_{3.5}\right)$ and $m\left(T_{4}\right)$ given in (2.10). The resulting value

$$
\hat{m}(\mathrm{Q}):=132.70453935
$$

agrees to ten significant figures with the DDM approximation (5.7), and to eight significant figures with the approximation (5.8) of Howell and Trefethen [7]. Unfortunately, however, our method of estimating the DDM errors cannot be applied to the decomposition of Fig. 5.4. More specifically, this method cannot be used to estimate the error in $\hat{m}(Q)$, because $m\left(Q_{12}\right)<3$ and, as a result, Theorem 3.1 cannot be used to estimate

$$
m\left(Q_{11,12,13}\right)+\left\{m\left(Q_{11,12}\right)+m\left(Q_{12,13}\right)-m\left(Q_{12}\right)\right\} .
$$

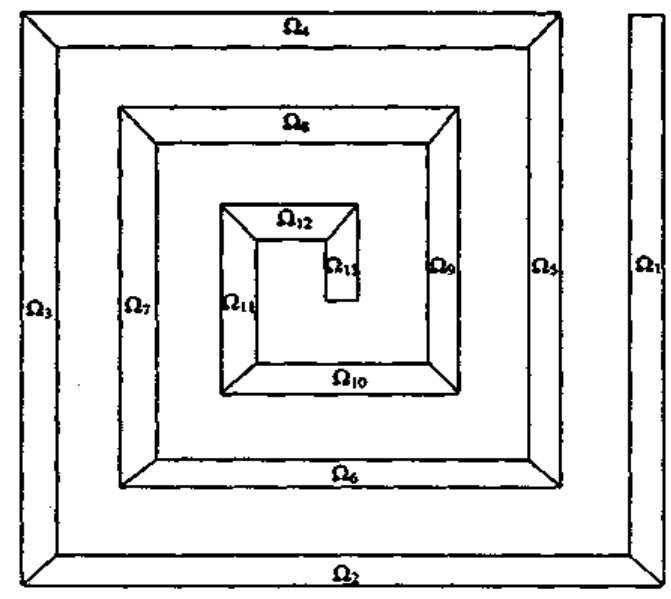

Figure 5.4 


\section{REFERENCES}

[1] F. Bowman, Introduction to Elliptic Functions (English University Press, London 1953 ).

[2] D. Gaier and W.K. Hayman, On the computation of modules of long quadrilaterals, Constr. Approx. (to appear).

[3] D. Gaierand W.K. Hayman, Modules of long quadrilaterals andthickringdomains, Rendiconti di Matematica Roma (to appear).

[4] D.GaierandN. Papamichael, On the comparison of two numerical methods for conformal mapping, IMA J. Numer. Anal. 7 (1987) 261-282.

[5] W.K.Hayman, Remarks on Ahlfors' distortion theorem, Quart. Appl. Math. Oxford Ser. 19 (1948) 33-53.

[6] P. Henrici,Applied and Computational Complex Analysis, Vol. III (Wiley, New York 1986 ).

[7] I.H.Howelland L.N.Trefethen,Amodified Schwarz-Christoffel transformation for elongated regions, SIAM J. Sci. Statist. Comput. 11 (1990) 928-949.

[8] N. Papamichael, Numerical conformal mapping onto a rectangle with applications to the solution of Laplacian problems, J. Comput. Apl. Math. 28 (1989) 63-83.

[9] N. Papamichael and N.S. Stylianopoulos, A domain decomposition method for conformal mapping onto a rectangle, Constr. Approx. (to appear).

[10] N.Papamichael and N.S.Stylianopoulos,Onthenumericalperformanceofa domain decomposition method for conformal mapping, pp. 155-169 of : St Ruscheweyh, E.B. Saff and R.S. Varga (Eds ), Computational Methods and Function Theory, Lecture Notes in Maths 1435 ( Springer-Verlag, Berlin 1990 ).

[11] L.N. Trefethen, SCPACKUser's Guide, Numerical Analysis Report 89-2, Dept of Maths Massachusetts Institute of Technology, Cambridge, MA, 1989. 


\section{NOT TO BE \\ REMOVED}

FROM THE LIBRARY 